\section{Jaw-dropping new surgical approach}

Scaffold-free tissue-engineered disc implants could be a viable early treatment option for degenerative pathologies of the temporomandibular joint (TMJ), according to new research published in Science Translational Medicine. Using a new surgical approach and animal model, the researchers demonstrate the safety and healing potential of such implants in early-stage TMJ disc disease.

Dysfunction of the TMJ is a common cause of pain and disability and can have multiple underlying causes (including injury and arthritis). Changes in the TMJ disc (such as disc thinning) are an early sign of TMJ degeneration and dysfunction. To address a lack of reparative treatments for TMJ pathologies, the authors investigated the use of scaffold-free

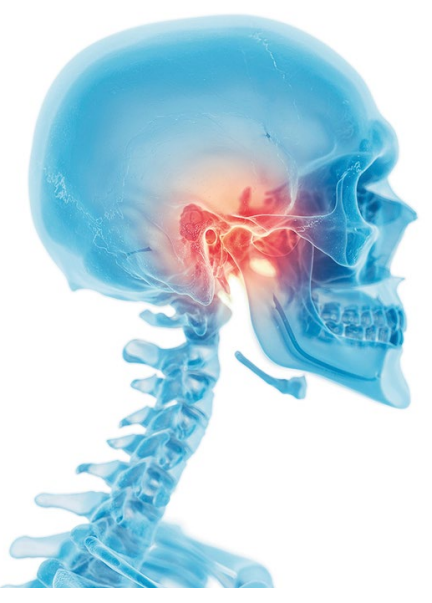

tissue-engineered implants for repairing disc thinning.

The researchers used rib cartilage cells to engineer scaffold-free allogeneic TMJ disc implants using a previously tested methodology. These implants had similar biomechanical and biochemical properties to native discs and were durable, safe and well-tolerated immunologically when implanted in Yucatan minipigs.

"To achieve secure fixation of the implant to a defect, we had to develop a novel surgical approach," states corresponding author Kyriacos Athanasiou. The surgery involved dissecting a horizontal pouch in the lateral region of the disc and removing a circular fenestration from the inferior lamina (mimicking disc thinning), creating two defects (a pouch and a fenestration). This approach provided biomechanical protection of the implant and enabled the researchers to test the reparative properties of the implant.

The implant prevented degenerative changes in the TMJ of minipigs and improved disc integrity as indicated by an increase in stiffness and closure of the TMJ disc defects compared with the empty defect (untreated) control.

"We are excited to see tissue engineering emerging as a viable strategy to address some of the most difficult musculoskeletal afflictions," states Athanasiou. "Eventually, it is our hope and objective that we will be able to move this work toward clinical use."

Jessica McHugh

ORIGINAL ARTICLE Vapniarsky, N. et al. Tissue engineering toward temporomandibular joint disc regeneration. Sci. Transl. Med. 10, eaaq1802 (2018)

\title{
Growth factors respond to cartilage damage
}

A study in Annals of the Rheumatic Diseases has identified an important function of connective tissue growth factor (CTGF) in response to mechanical stress and cartilage damage typically associated with osteoarthritis. "We have uncovered what we think may be the principal mechanism of action of CTGF and demonstrate a new mechanism by which TGF $\beta$ is activated in response to tissue injury," says corresponding author Tonia Vincent.

The pericellular matrix (PCM) surrounds and isolates chondrocytes from the stiffer extracellular matrix (ECM), and is thereby thought to be highly responsive to mechanical force. "We have previously shown that an important mechanism by which cartilage responds to sterile mechanical injury is by liberating growth factors bound to heparan sulphate in the PCM," notes Vincent. Her group has worked extensively on one of the best characterized of these responses, the release and activation of fibroblast-like growth factor 2 .

In the new study, the researchers detect CTGF in the PCM of human articular cartilage and its release into culture medium upon incision damage to explants. They also present microarray evidence that stimulation of chondrocytes with recombinant CTGF induces genes involved in canonical TGF $\beta$ signalling. Importantly, gel comigration and co-immunoprecipitation data show that CTGF interacts covalently with TGF $\beta$, functioning as a binding protein to sequester latent TGF $\beta$ in the PCM until its release on injury. Subsequent activation of this complex, the researchers believe, results from the complex binding to TGF $\beta$ receptor 3 on the chondrocyte.

The proposed function of CTGF is also supported by in vivo data. Vincent's team found that less TGF $\beta$ was released in response to hip avulsion (to model cartilage injury) of mice with

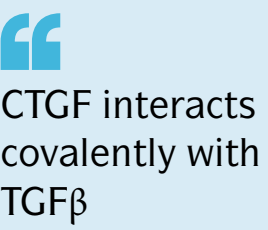

- Mechanical stress

- Cartilage injury
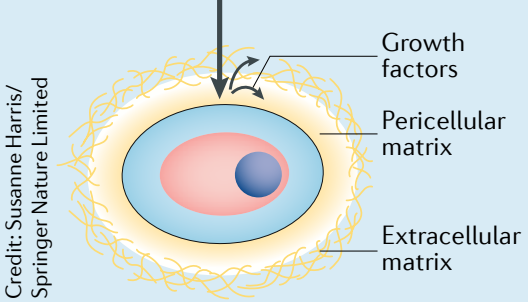

tamoxifen-induced knockout of floxed Ctgf, compared with floxed mice not treated with tamoxifen as a control.

However, unexpectedly, the

Ctgf-deleted mice were more resistant to destabilization of the medial meniscus (osteoarthritis model), an effect attributable to increased thickness of the cartilage associated with constitutively active SMAD2.

To explain this surprising phenotype further data are needed; Vincent speculates that this effect may be due to over-compensation by other TGF $\beta$ family members.

Nicholas J. Bernard

ORIGINAL ARTICLE Tang, X. et al. Connective tissue growth factor contributes to joint homeostasis and osteoarthritis severity by controlling the matrix sequestration and activation of latent TGF $\beta$. Ann. Rheum. Dis. https://doi. org/10.1136/annrheumdis-2018-212964 (2018) 